\title{
An evaluation of the role of tumor load in cytoreductive nephrectomy
}

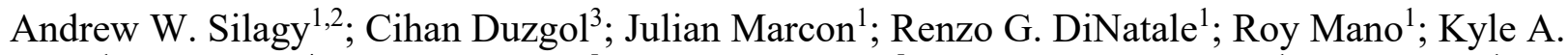
Blum ${ }^{1}$; Ed Reznik ${ }^{4}$; Martin H. Voss ${ }^{5}$; Robert J. Motzer ${ }^{5}$; Jonathan A. Coleman ${ }^{1}$; Paul Russo ${ }^{1}$; Oguz Akin ${ }^{3}$; A. Ari Hakimi ${ }^{1}$

${ }^{1}$ Urology Service, Department of Surgery, Memorial Sloan Kettering Cancer Center, New York, NY, United States; ${ }^{2}$ Department of Surgery, University of Melbourne, Austin Hospital, Melbourne, Australia; ${ }^{3}$ Department of Radiology, Memorial Sloan Kettering Cancer Center, New York, NY, United States; ${ }^{4}$ Department of Epidemiology and Biostatistics, Memorial Sloan Kettering Cancer Center, New York, NY, United States; ${ }^{5}$ Department of Medicine, Memorial Sloan Kettering Cancer Center, New York, NY, United States

Funding: This research was funded in part through the NIH/NCI Cancer Center Support Grant P30 CA008748.

Cite as: Can Urol Assoc J 2020 June 16; Epub ahead of print. http://dx.doi.org/10.5489/cuaj.6350

Published online June 16, 2020

$* * *$

\section{Abstract}

Introduction: New radiological tools can accurately provide preoperative three-dimensional spatial assessment of metastatic renal cell carcinoma (RCC) We aimed to determine whether the distribution, volume, shape, and fraction of RCC resected in a cytoreductive nephrectomy associates with survival.

Methods: We retrospectively reviewed 560 patients undergoing cytoreductive nephrectomy performing a comprehensive volumetric analysis in eligible patients of all detectable primary and metastatic RCC prior to surgery. We used Cox regression analysis to determine the association between the volume, shape, fraction resected, and distribution of RCC and overall survival (OS). Results: There were 62 patients eligible for volumetric analysis, with similar baseline characteristics to the entire cohort, and median survivor followup was 34 months. Larger primary tumors were less spherical, but not associated with different metastatic patterns. Increased primary tumor volume and tumor size, but not the fraction of tumor resected, were associated with inferior survival. The rank of tumors based on unidimensional size did not completely correspond to the rank by primary tumor volume, however, both measurements yielded similar concordance for predicted OS. Larger tumor volume was not associated with a longer postoperative time off treatment.

Conclusions: Primary tumor volume was significant for predicting OS, while the fraction of disease resected did not appear to impact upon patient outcomes. Although rich in detail, our 
study is potentially limited by selection bias. Future temporal studies may help elucidate whether the primary tumor shape is associated with tumor growth kinetics.

\section{Introduction}

In 2019 in the United States, kidney cancer is estimated to cause around 14,770 deaths. ${ }^{1}$ Upfront surgery is recommended to reduce mortality from localized disease, however, when renal cell carcinoma (RCC) has already metastasized, the role of surgery is not definitive. The CARMENA trial reported non-inferiority for upfront Sunitinib compared with cytoreductive surgery followed by systemic treatment. ${ }^{2}$ However, the studies' principal investigators excluded some patients with a low metastatic burden "at the investigator's discretion" and did not report on whether the fraction of tumor removed during nephrectomy, or the location of metastases, were associated with outcome.

We have previously reported that primary tumor size is an independent predictor of survival following cytoreductive nephrectomy. ${ }^{3}$ However, this finding did not consider that tumor volume can vary, even in tumors with identical maximum axial diameter, depending on the shape of disease. ${ }^{4}$ Previous studies by Pierorazio et al and Barbastefano et al showed that the fraction of disease resected in a cytoreductive nephrectomy may be significant for survival outcomes, with improved outcomes when a minimum of $90-95 \%$ of all disease is debulked. 5,6

Therefore, we aimed to test the findings described by Pierorazio et al and Barbastefano et al in an independent cohort, using modern computed tomography (CT) software tools. Further, we aimed to identify how the volume, shape and distribution of disease varies and whether this variation relates to survival after cytoreductive nephrectomy.

\section{Methods}

\section{Patients}

After obtaining institutional review board approval, we reviewed 560 patients from our prospectively maintained nephrectomy database with metastatic disease treated with a cytoreductive nephrectomy. As part of a collaborative effort, patients with clear cell histology and next generation sequencing of tumors were retrospectively evaluated for total volumetric analysis of all detectable disease. This required patients to have a CT scan prior to surgery of the chest, abdomen and pelvis, plus additional peripheral CT imaging if other distant metastases were suspected. Patients needed to have non-contrast and nephrographic phase studies available for volumetric analysis. There were 62 patients that met all inclusion criteria and their baseline clinicopathological variables, including International Metastatic Renal-Cell Carcinoma Database Consortium (IMDC) risk, ${ }^{7}$ and time to systemic treatment were recorded for analysis. 


\section{Image analysis}

Primary and metastatic disease was measured using TeraRecon semi-automated segmentation analysis. ${ }^{8}$ A systematic approach was taken to individually count and measure every single detectable metastasis. This process was overseen by a radiologist (CD), specializing in wholebody CT analysis. Outputs from this segmentation analysis included RECIST 1.1 scores, volume calculations and tumor compactness measurement (an assessment of sphericity). Binary observations were made of whether the primary tumor contours were well-defined radiographically.

\section{Statistical analysis}

We visually reviewed the volume of disease and distribution of disease in patients prior to undergoing cytoreductive nephrectomy. We divided the volume of primary disease by the sum volumes of all measurable disease, including the primary, to determine the fractional percentage of tumor volume. The distribution volume was visualized and transformed to normalize the data. The Kaplan-Meier and Cox regression methods were used to determine whether the volume and the fraction of disease resected was associated with a difference in overall survival, both as a continuous variable and with the previously published cutoffs of $90 \%$ and $95 \%$ resection. The primary end point was overall survival following surgery, with patients alive at last follow-up date censored. We tested the shape of primary tumors to further determine whether irregularly shaped tumors were associated with different radiographic features, metastatic distributions and poorer survival outcomes. We further evaluated whether increased tumor volume was associated with longer time to commencing systemic therapy. Finally, to estimate generalizability, we compared the cohort's baseline characteristics with the clear cell histology cases that did not meet the selection criteria. A p-value $<0.05$ was considered to be statistically significant. All statistical analyses were performed using R Version 3.5.1. ${ }^{9}$

\section{Results}

The cohort consisted of 62 patients, 46/62 were male with a median age of 57 years old. Table 1 describes the cohort characteristics including the preoperative radiographic descriptive characteristics. Patients included in the study were slightly younger than the original cytoreductive nephrectomy cohort, with all other patient and tumor features balanced (Supplementary Table 1).

The median volume of primary tumors (PV) was $225 \mathrm{~cm}^{3}$, this was non-normally distributed, with a negative skew $(\mathrm{p}=0.007)$. The median total volume of disease (TV) was $236 \mathrm{~cm}^{3}$, with patients' TV predominantly restricted to the primary tumor (Figure 1). Only three tumors had a volume of metastatic disease (MV) greater than PV, the median percentage of TV resected was $96.7 \%$. Shape analysis was performed on the primary tumors, with five tumors unsuccessfully processed, due to small size (mean volume $27 \mathrm{~cm}^{3}$ ). There was an inverse correlation between PV and sphericity with larger PV being less compact (Spearman rank -0.26 , 
$\mathrm{p}=0.049)$. However, there was no association between the sphericity of the primary tumor and ill-defined tumor borders, the number of metastases or the volume of metastatic disease.

The long axis size on CT was concordant with the final pathological specimen diameter, with a median difference between the pathological tumor size and radiographic size of $3.6 \mathrm{~mm}$ (mean $2 \mathrm{~mm}$ ). Interestingly, while the rank of tumors measuring maximal unidimensional CT size was generally comparable to the rank based on PV, some tumors moved significantly in their rank when switching between volume and unidimensional size measurement (Figure 2). The median number of metastatic organs involved was two and the median number of measurable disease sites at the time of resection (including the primary) was 3.5. Over half of all patients had metastatic disease in their lung, however, only just over one quarter had the largest volume of their disease in the lung, with axial and appendicular skeletal sites representing the largest combined site for the highest volume metastatic deposits (Table 2).

There were 52 patients that received systemic therapy after cytoreduction, with a median time from surgery to commencing systemic therapy of 88 days. Of note, $26 / 62$ patients underwent a period of close postoperative active surveillance, with either minimal residual disease or no evidence of disease, with eight patients remaining off-treatment at last follow-up. Active surveillance was associated with a $\operatorname{lower} \log _{10}(\mathrm{PV})(\mathrm{p}<0.001)$. Although larger primary volume was associated with a shorter time to systemic therapy, when you remove the patients that underwent a period of active surveillance, there was no association between primary tumor volume and time to treatment (Figure 3).

There were 29 deaths during the follow-up period, with a median follow-up time of 34 months. Median survival was 4.48 years from surgery. When grouping the fraction of TV resected by published criteria $(<90 \%, 90-95 \%$ and $>95 \%)$ or as a continuous variable there was no significant difference in survival. A $\log _{10}$ transformation of the $\mathrm{PV}$ produced a similar hazard ratio and $\mathrm{p}$-value for survival (HR 2.62, 95\% CI $1.23-5.62, \mathrm{p}=0.01$ ) compared with radiographic long axis length (HR $1.16,95 \% \mathrm{CI} 1.04-1.30, \mathrm{p}=0.007)$. This similarity persisted when adjusting for sphericity and IMDC risk stratification ( $\mathrm{p}=0.049$ and $\mathrm{p}=0.016$, respectively). An increase in $\log _{10}(\mathrm{TV})$ also corresponded with inferior survival $(\mathrm{p}=0.02)$, however the sphericity of the primary tumor, number of metastatic lesions and number of organs harboring those metastases were not significant for overall survival. Overall, unidimensional CT size, $\log _{10}(\mathrm{PV})$ and $\log _{10}(\mathrm{TV})$ yielded similar concordance with a c-index of $0.674,0.666,0.655$, respectively.

\section{Discussion}

This study presents an in-depth analysis of the volume and distribution of RCC at the time of undergoing cytoreductive nephrectomy and their corresponding impact on postoperative survival. We identified that in patients selected for cytoreductive nephrectomy, an increase in the $\log 10(\mathrm{PV})$ was associated with worse overall survival and that this impact was comparable to an increase in the radiographic long axis size of the primary tumor. 
We analyzed the distribution of disease and saw that patients selected for surgery predominantly had disease contained to their primary tumor. The most common sites of metastases were lung, supradiaphragmatic nodes and regional nodes $(55 \%, 26 \%, 24 \%$, respectively) and the most common sites containing the largest volume of metastases were lung, vertebra and appendicular bone $(27 \%, 15 \%, 11 \%$, respectively). Another notable finding was that larger tumors were less spherical, although the change in shape alone was not associated with inferior survival. Future longitudinal exploration could explore whether the architectural tumor shape changes over time.

Although the fraction of TV resected was not associated with survival in our cohort, there appeared to be a significant impact from PV. De Bruijn et al looked at patients with intermediate-risk IMDC profile and did not find an independent association between either baseline tumor volume or fraction resected and overall survival. ${ }^{10}$ Notably, the majority of patients in our cohort were intermediate-risk further reinforcing our finding that the fraction of disease resected is probably not an effective measurement for predicting survival outcomes after cytoreductive nephrectomy. Interestingly, in our cohort, it was the patients with a lower TV of disease that harbored higher proportions of MV. This is probably due to selection bias and ascertainment bias, whereby patients with a high MV and TV are assessed by surgeons as unsuitable candidates for cytoreductive nephrectomy and a higher proportion of these patients are deceased. A potential confounder to the contention that higher PV confers an adverse prognosis after cytoreductive nephrectomy is that recovery time following resection of a larger tumor may be longer. While patients undergoing a period of postoperative active surveillance after surgery had a lower PV, among patients planned for systemic therapy immediately after surgery, there was no correlation between $\log _{10}(\mathrm{PV})$ and time to initiating systemic therapy. Given the similar postoperative recovery times, the worse hazard for increasing $\log _{10}(\mathrm{PV})$ potentially represents a more aggressive phenotype. Despite a quarter of our cohort consisting of high-risk IMDC patients, overall survival across the entire cohort was comparable to the expected survival for IMDC intermediate-risk patients. This result is likely due to surgeons selecting patients that they deem to be most likely to benefit from cytoreductive nephrectomy.

While we could not reproduce the previous finding that the fraction of TV resected is associated with survival, this may be due to methodological factors, along with differences in patient selection. Disease volume can be calculated radiographically by extrapolating from unidimensional or bidimensional measurements, or using segmentation software. Unlike unidimensional measurements, segmentation estimates can account for irregularly shaped tumors, whereas maximal axial size measurements may lead to an overestimation of volume. ${ }^{11}$ In this small cohort, due to the variation in tumor sphericity, multiple tumors shifted in size rank when ordered by unidimensional size compared with volume, although most did not change markedly. This is particularly important for assessing correlations for populations with nonparametric distributions. Therefore, estimating volume measurements from long axis size 
measurements will result in a different order of tumors volumes compared with rankings from segmentation analysis. Blute et al similarly illustrated that unidimensional measurements were unsuitable for calculating volume, this was not linked to differences between sphericity. ${ }^{12}$ Nonetheless, our findings demonstrated a strong concordance between both increases in $\log _{10}(\mathrm{PV})$ and unidimensional size measurements with overall survival.

Our cohort has limitations due to selection biases by both surgical case selection and strict eligibility for volumetric analysis. The data benefits from in-depth analysis of volume of disease, however, as a small non-consecutive case series, larger populations may demonstrate different overall survival outcomes. Reassuringly, aside from a slightly younger population, the clinicopathological characteristics of this series largely reflected the profile of the institution's broader cytoreductive nephrectomy experience. The study provides a thorough snapshot of disease status immediately prior to surgery, although we do not capture temporal factors such as the rate of primary tumor growth and the rate of progression of metastatic disease. Nonetheless, the relatively small case numbers warrant caution with interpretation and the novel findings outlined require external validation.

While this project provides considerable biological insights into the volumetric patterns of metastatic RCC, the process of obtaining the data, using semi-automated segmentation remains time-consuming. Given the similarity in predicted outcomes for both volume and long axis measurement, unidimensional size serves as an effective surrogate for tumor volume, therefore it is unlikely that volumetry would significantly improve patient selection and prognostication. However, with the growth of both artificial intelligence and automated radiological analysis, surgical decision-making in the future may ultimately integrate tumor segmentation. ${ }^{13,14}$

\section{Conclusions}

An increasing $\log _{10} \mathrm{PV}$ is associated with reduced overall survival and less-spherical primary tumors. Future studies could identify whether sphericity changes depending on tumor growth kinetics and whether it is associated with distinct mutational profiles. 


\section{References}

1. Siegel, R. L., Miller, K. D., Jemal, A.: Cancer statistics, 2019. CA Cancer J Clin, 69: 7, 2019

2. Méjean, A., Ravaud, A., Thezenas, S. et al.: Sunitinib Alone or after Nephrectomy in Metastatic Renal-Cell Carcinoma. New England Journal of Medicine, 379: 417, 2018

3. DiNatale, R. G., Xie, W., Becerra, M. F. et al.: The Association Between Small Primary Tumor Size and Prognosis in Metastatic Renal Cell Carcinoma: Insights from Two Independent Cohorts of Patients Who Underwent Cytoreductive Nephrectomy. Eur Urol Oncol, 2019

4. Laubender, R. P., Lynghjem, J., D’Anastasi, M. et al.: Evaluating the agreement between tumour volumetry and the estimated volumes of tumour lesions using an algorithm. European Radiology, 24: 1521, 2014

5. Pierorazio, P. M., McKiernan, J. M., McCann, T. R. et al.: Outcome after cytoreductive nephrectomy for metastatic renal cell carcinoma is predicted by fractional percentage of tumour volume removed. BJU International, 100: 755, 2007

6. Barbastefano, J., Garcia, J. A., Elson, P. et al.: Association of percentage of tumour burden removed with debulking nephrectomy and progression-free survival in patients with metastatic renal cell carcinoma treated with vascular endothelial growth factortargeted therapy. BJU Int, 106: 1266, 2010

7. Heng, D. Y. C., Xie, W., Regan, M. M. et al.: External validation and comparison with other models of the International Metastatic Renal-Cell Carcinoma Database Consortium prognostic model: a population-based study. The Lancet. Oncology, 14: 141, 2013

8. Lodewick, T. M., Arnoldussen, C. W. K. P., Lahaye, M. J. et al.: Fast and accurate liver volumetry prior to hepatectomy. HPB : the official journal of the International Hepato Pancreato Biliary Association, 18: 764, 2016

9. R Core Team: R: A language and environment for statistical computing. Vienna, Austria: R Foundation for Statistical Computing, 2018

10. de Bruijn, R. E., Nijkamp, J., Noe, A. et al.: Baseline tumor volume in assessing prognosis of patients with intermediate-risk synchronous metastatic renal cell carcinoma. Urologic Oncology: Seminars and Original Investigations, 34: 258.e 7, 2016

11. Welsh, J. L., Bodeker, K., Fallon, E. et al.: Comparison of response evaluation criteria in solid tumors with volumetric measurements for estimation of tumor burden in pancreatic adenocarcinoma and hepatocellular carcinoma. The American Journal of Surgery, 204: 580,2012

12. Blute, M. L., Jr., Ziemlewicz, T. J., Lang, J. M. et al.: Metastatic Tumor Burden Does Not Predict Overall Survival Following Cytoreductive Nephrectomy for Renal Cell Carcinoma: a Novel 3-Dimensional Volumetric Analysis. Urology, 100: 139, 2017

13. Zhang, G. M. Y., Shi, B., Xue, H. D. et al.: Can quantitative CT texture analysis be used to differentiate subtypes of renal cell carcinoma? Clinical Radiology, 74: 287, 2019

14. Bier, G., Bier, S., Bongers, M. N. et al.: Value of computed tomography texture analysis for prediction of perioperative complications during laparoscopic partial nephrectomy in patients with renal cell carcinoma. PLOS ONE, 13: e0195270, 2018 


\section{Figures and Tables}

Fig. 1. Distribution of tumor volume.

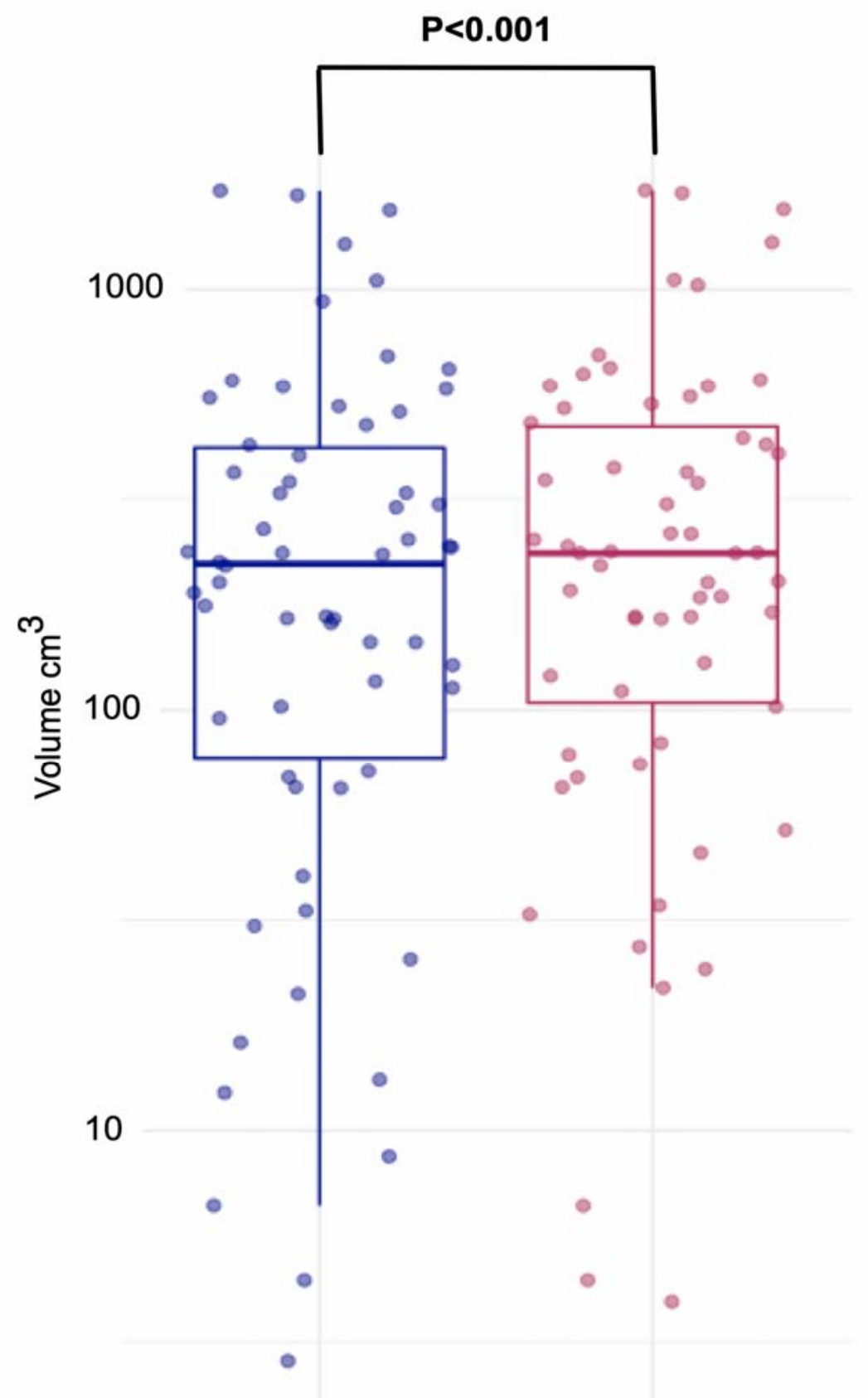

Primary Volume (PV) Total Volume (TV) 
Fig. 2. Order of patients by tumor size.

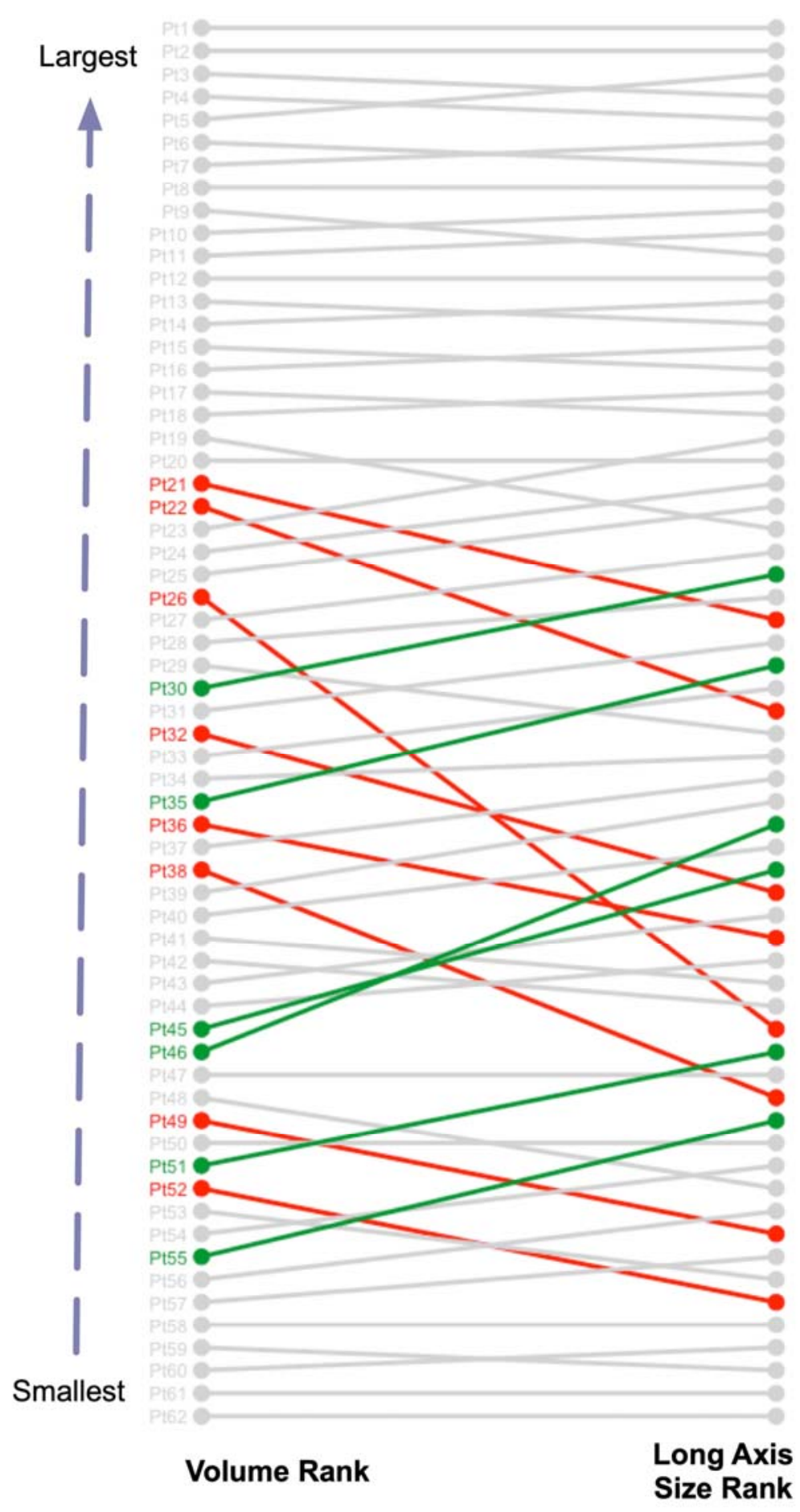

Significant fall Significant rise Relatively stable 
Fig. 3. Primary volume and time to treatment.

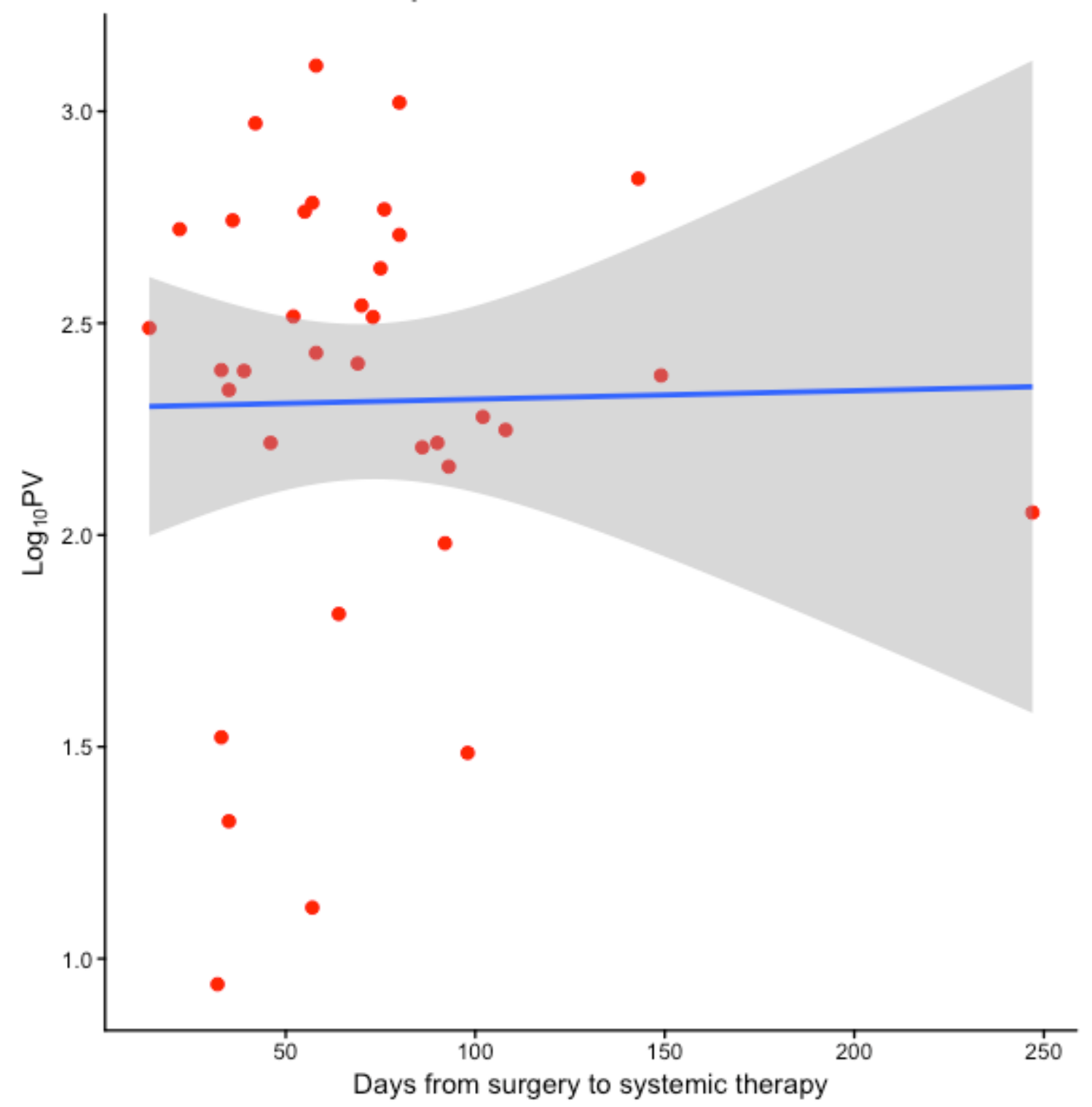




\begin{tabular}{|c|c|}
\hline \multicolumn{2}{|l|}{ Table 1. Cohort characteristics } \\
\hline Cohort & 62 \\
\hline Male gender $(\%)$ & $46(74.2)$ \\
\hline Age, median (IQR) & $57.47(52.52,63.30)$ \\
\hline Tumor size $(\mathrm{cm})$, median $(\mathrm{IQR})$ & $7.90(5.62,10.17)$ \\
\hline $\begin{array}{l}\text { Pathological vs. radiographic size difference }(\mathrm{mm}) \text {, mediar } \\
\text { (IQR) }\end{array}$ & $-0.36(-1.18,0.45)$ \\
\hline \multicolumn{2}{|l|}{ Tumor stage $(\%)$} \\
\hline T0 & $1(1.6)$ \\
\hline $\mathrm{T} 1$ & $6(9.7)$ \\
\hline $\mathrm{T} 2$ & $11(16.1)$ \\
\hline T3 & $46(74.2)$ \\
\hline T4 & $6(9.7)$ \\
\hline Node-positive (\%) & $24(38.7)$ \\
\hline \multicolumn{2}{|l|}{ IMDC risk group } \\
\hline High-risk & $16(25.8)$ \\
\hline Intermediate-risk & $43(69.4)$ \\
\hline Low-risk & $2(3.2)$ \\
\hline Unknown & $1(1.6)$ \\
\hline Radiographic tumor contours ill-defined (\%) & $38(61.3)$ \\
\hline Number of lesions, metastatic and primary, median (IQR) & $3.50(2.00,5.00)$ \\
\hline Number of metastatic organs involved, median (IQR) & $2.00(1.00,2.00)$ \\
\hline
\end{tabular}

IMDC: International Metastatic Renal Cell Carcinoma Database Consortium; IQR: interquartile range. 


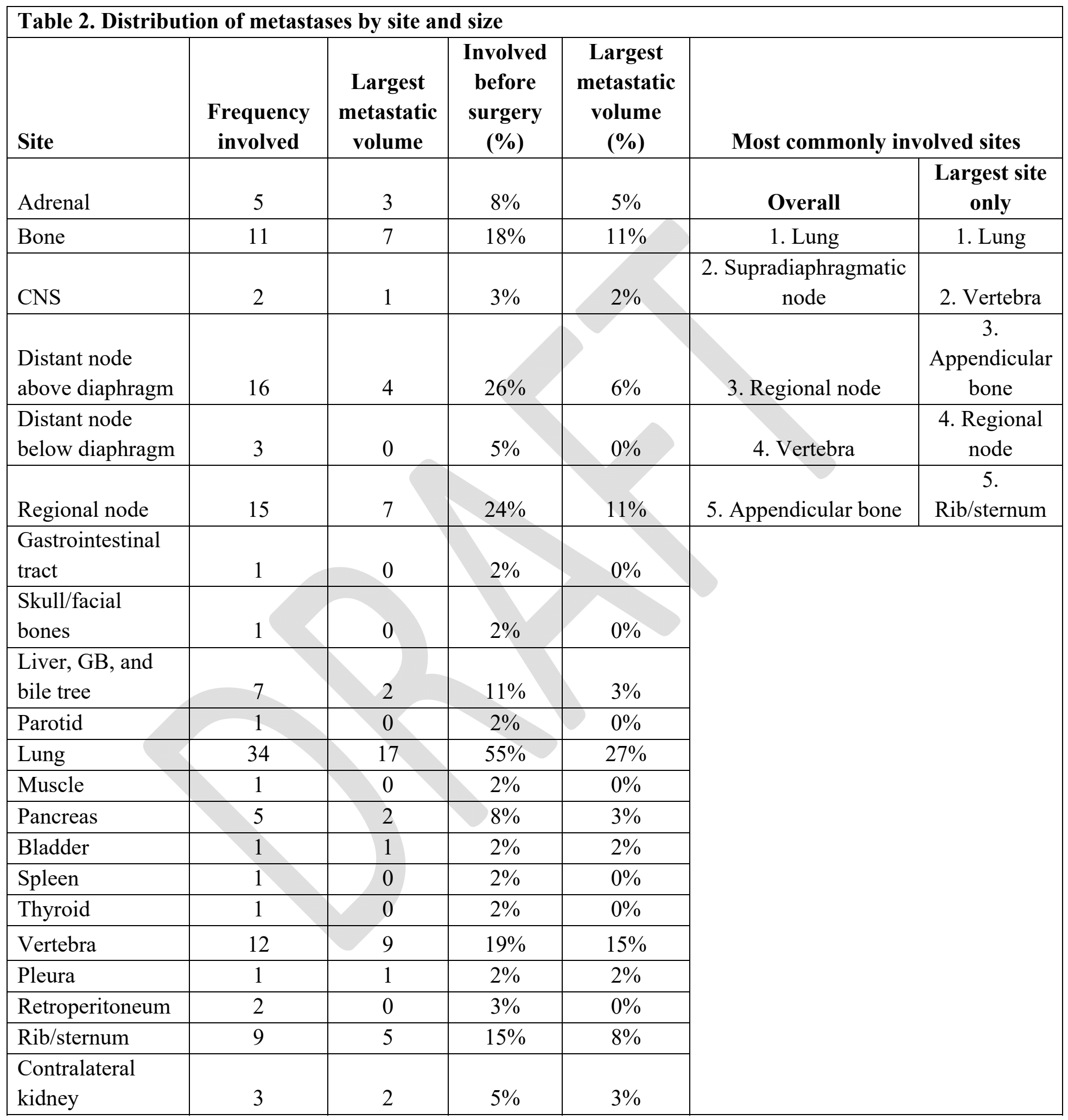

CNS: central nervous system; GB: gall bladder. 


\begin{tabular}{|l|c|c|c|}
\hline \multicolumn{5}{|c|}{ Supplemental Table 1. Baseline characteristics of included and excluded patients } \\
\hline & Remaining cohort & Volumetric study & p \\
\hline Number & 368 & 62 & \\
\hline Male gender (\%) & $274(74.5)$ & $46(74.2)$ & 1 \\
\hline Age, median (IQR) & $61.67(52.96,68.56)$ & $57.47(52.52,63.30)$ & 0.022 \\
\hline Size, median (IQR) & $8.85(6.50,11.00)$ & $7.90(5.62,10.17)$ & 0.318 \\
\hline pT3/4 (\%) & $292(80.0)$ & $52(85.2)$ & 0.385 \\
\hline Nodal metastases (\%) & $102(27.7)$ & $24(38.7)$ & 0.096 \\
\hline Bone metastases (\%) & $116(31.5)$ & $24(38.7)$ & 0.305 \\
\hline Visceral metastases (\%) & $283(76.9)$ & $44(71.0)$ & 0.335 \\
\hline Other metastases (\%) & $38(10.3)$ & $5(8.1)$ & 0.819 \\
\hline Number of metastatic sites (\%) & & & 0.092 \\
\hline 1 & $195(53.0)$ & $24(38.7)$ & \\
\hline 2 & $103(28.0)$ & $21(33.9)$ & \\
\hline$>2$ & $70(19.0)$ & $17(27.4)$ & \\
\hline
\end{tabular}

